\title{
Deformation of the lamina cribrosa by elevated intraocular pressure
}

\author{
D B Yan, F M Coloma, A Metheetrairut, G E Trope, J G Heathcote, C R Ethier
}

\begin{abstract}
The purpose of this study was to determine the mechanical response of the lamina cribrosa (LC) to elevated intraocular pressure (IOP) so as to identify possible mechanisms of optic nerve damage in early glaucoma. Ten pairs of normal human eyes were fixed after 24 hours' exposure to $50 \mathrm{~mm} \mathrm{Hg}$ pressure (experimental eyes) or $5 \mathrm{~mm} \mathrm{Hg}$ pressure (contralateral control eyes). Photomicrographs of the central region of the optic nerve head (ONH) were taken to examine the LC morphologically and to measure the dimensions of the LC. It was found that elevated IOP caused the LC to deflect posteriorly without affecting its thickness. The majority of the posterior displacement in the LC occurred near the periphery of the ONH. This shape change is consistent with a model of force distribution within the $L C$ in which shear stresses are dominant; such stresses are maximal at the periphery and minimal at the centre of the ONH. These findings support a model in which mechanical forces, specifically shearing stresses within the peripheral lamina, play a direct role in the pathology of glaucomatous optic neuropathy. (BrF Ophthalmol 1994; 78: 643-648)
\end{abstract}

Visual field loss and disc cupping characteristic of primary open angle glaucoma are thought to be due to optic nerve fibre damage at the level of the lamina cribrosa (LC). Since it is known that elevated intraocular pressure (IOP) can disrupt the structural organisation of the $\mathrm{LC}^{1-3}$ and interrupt axoplasmic flow at the level of the $\mathrm{LC},{ }^{4 \rightarrow}$ it has been previously postulated by numerous investigators (for example, Maumenee $^{8}$ ) that the axonal damage in glaucoma is due to mechanical stresses within the LC acting upon the nerve bundles. To evaluate this hypothesis, it is essential to understand the mechanical response of the LC to elevated IOP.

Quigley $^{3}$ examined the LC in normal and glaucomatous eyes fixed by immersion (at zero pressure) and correlated structural alterations to the LC with the clinical severity of the glaucoma. Zeimer and Ogura' and Coleman et $a l^{10}$ indirectly determined changes in the position of the LC by measuring the location of the anterior face of the optic nerve head (ONH) in normal and glaucomatous eyes when IOP was raised or lowered, thereby making inferences about the effective rigidity of the LC. Levy and coworkers $^{11}{ }^{12}$ examined retrodisplacement of the LC in human and primate eyes as a result of elevated IOP by tracking the position of a platinum wire embedded in the LC. They found that the LC retrodisplaced minimally adjacent to the sclera and maximally at the centre, conclud- ing that LC displacement could cause shearing of $\mathrm{ONH}$ axons owing to differential displacement of the connective tissue. Nesterov and Egorov ${ }^{13}$ fixed human eyes at 15 and $45 \mathrm{~mm} \mathrm{Hg}$ and observed that the mean distance from the anterior retinal surface to the posterior surface of the LC was greater in high pressure eyes.

In this paper, we seek to complement previous work by a direct morphometric study of the response of the LC in normal human eyes to acute changes in IOP. We feel that this approach has several advantages. Firstly, a morphometric approach allows both the overall shape and the internal architecture of the LC to be evaluated as a function of pressure. This is in contrast with previous studies of the effects of pressure on the LC which focused on positional changes of the anterior surface of the retina at the $\mathrm{ONH}$ or on the topography of the ONH. Secondly, the use of normal eyes in acute experiments permits potential assessment of the factors initiating axonal damage, since the effect of IOP on a presumably normal ONH can be studied. This is important since $\mathrm{ONH}$ architecture in glaucomatous eyes will be substantially different from that of normal eyes, owing to degenerative optic neuropathy.

The goals of the present work were: (i) to characterise and quantify acute deformations of the LC as a result of elevated IOP; (ii) to formulate a mechanical model of the stress distribution within the LC which is consistent with clinically and experimentally observed patterns of axonal damage in glaucoma; and (iii) to identify features of the LC which might increase the susceptibility of some eyes to glaucomatous damage. The experimental approach was to fix normal human eyes at either high or low pressure and then to compare morphometrically the LC from the low and high pressure groups.

\section{Materials and methods}

Ten pairs of ostensibly normal human eyes (average age 69 years) from which the corneas had been removed for transplantation were obtained within 24 hours post mortem from the Eye Bank of Ontario. The eyes were hemisected at the equator and the vitreous was carefully removed under an operating microscope. This dissection was performed while suspending the eye in a saline bath to prevent the retina from detaching. Each posterior hemisphere was then mounted onto a plastic perfusion dish ${ }^{14}$ which sealed it at the equator and allowed it to be pressurised. Briefly, this dish had a small discshaped elevated region with bevelled edges which effectively matched the inner diameter of the globe at the equator. The eye was then clamped in position by a ring whose inner 
diameter matched the outer diameter of the globe at the equator. This ring was secured by screws, resulting in a fluid-tight seal at the equator. The dish was equipped with ports for fluid so that the pressure within the globe could be controlled. Clamping of the globe at the equator produced essentially no distortion at the optic nerve head, since the clamping location was well removed from the area of interest and minimally deformed the scleral shell. One eye was pressurised to $50 \mathrm{~mm} \mathrm{Hg}$ while the contralateral eye was pressurised to $5 \mathrm{~mm} \mathrm{Hg}$ using Dulbecco's phosphate buffered saline (DPBS) with $5.5 \mathrm{mM}$ glucose. The high and low pressures of 5 and $50 \mathrm{~mm} \mathrm{Hg}$ were chosen to span the extremes of what may be encountered clinically. In selecting these pressures our primary motivation was to produce significant differences in displacement which could be easily identified and quantified in a preliminary study.

After 24 hours under pressure at $4^{\circ} \mathrm{C}$, the DPBS solution was exchanged with fixative ( $2.5 \%$ paraformaldehyde in Sörenson's phosphate buffer) while maintaining the pressure. The eyes were fixed at pressure in this manner for 24 hours, and then the $\mathrm{ONH}$ with surrounding sclera was excised in such a way as to preserve ONH orientation. The tissue segment was dehydrated in a graded series of ethanols, then infiltrated and embedded with JB-4 plastic (Polysciences Inc, Warrington, PA, USA). Serial sections, sagittally oriented, were cut so that a $4 \mu \mathrm{m}$ thick section was obtained at $100 \mu \mathrm{m}$ intervals across the $\mathrm{ONH}$. For these initial morphometric studies, one section per eye which was free from technical artefacts was selected from near the centre of the $\mathrm{ONH}$ (typically within $150 \mu \mathrm{m}$ of the centre, but not including the central retinal artery). The centre of the $\mathrm{ONH}$ was taken to be the section having the largest scleral canal diameter. Sections were stained with a Trichrome stain (Gill's haematoxylin, EM Diagnostics Systems Inc, Gibbstown, NJ, USA; Biebrich scarlet, acid fuchsin and aniline blue, BDH Inc, Toronto, Canada) using a microwave method as described by Maynard, ${ }^{15}$ and sections for morphometry were photographed (final magnification $\times 107$ ).

In addition to qualitative comparisons of the LC structure in eyes fixed at low and high pressures, a morphometric study was undertaken. The $\mathrm{LC}$ was identified as a prominent blue staining band of connective tissue, with anterior and posterior limits defined to be the zones where the tissue consisted of approximately half neural tissue and half connective tissue. Using this definition, the anterior and posterior limits of the LC were independently marked on each photo-

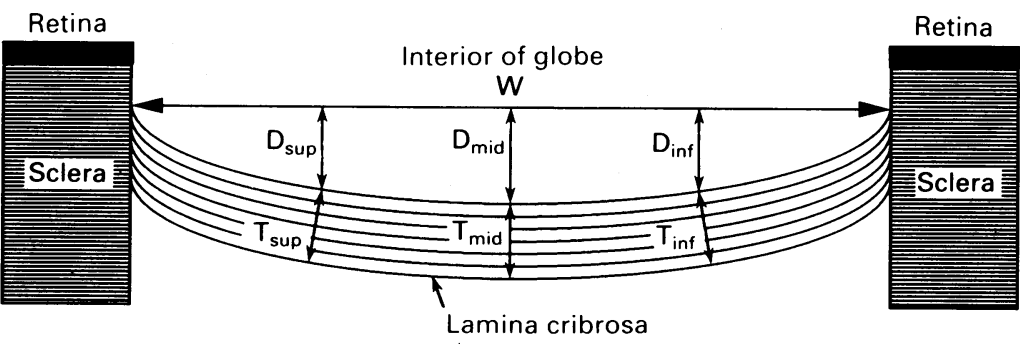

Figure 1 Schematic view of the lamina cribrosa (solid lines) showing surrounding sclera overlain by retina. Definitions of the measured quantities width (W), posterior displacement (D), and thickness $(T)$ are also shown. micrograph by three observers blinded to the fixation pressure and eye pairings. Based on these limits, the width (W), posterior displacement (D), and thickness (T) of the LC were measured from micrographs. The width was taken to be the distance between the edges of the sclera at the anterior limit of the LC (Fig 1). The posterior displacement was taken to be the perpendicular distance from the line used to measure the width to the anterior limit of LC, while the thickness was taken to be the perpendicular distance from the anterior to the posterior limit of the LC. Thickness and posterior displacement were measured with a ruler at three points equidistantly spaced across the width of the LC, corresponding to the anatomical midpoint (mid), superior point (sup), and inferior point (inf) along the central sagittal section. The three thickness values $\left(T_{\text {mid }}, T_{\text {sup }}, T_{\text {inf }}\right)$ were averaged to obtain average $L C$ thickness $\left(T_{\text {avg }}\right)$. The posterior displacement at the midpoint $\left(\mathrm{D}_{\text {mid }}\right)$ was divided by $\mathrm{W}$ to obtain a normalised midpoint posterior displacement for each eye. Since this quantity has been normalised by LC width, it should be essentially independent of the actual size of the $\mathrm{ONH}$, and should instead depend primarily on the shape of the LC. Specifically, $\mathrm{D}_{\text {mid }} / \mathrm{W}$ is a simple normalised measure which is representative of the posterior bowing or curvature of the anterior surface of the LC. Finally, an estimate of the percentage of the total posterior displacement occurring in the periphery of the LC, $\%_{\text {sup }}$ and $\%_{\text {inf }}$, was obtained by dividing the change in posterior displacement at the periphery $\left(\Delta D_{\text {sup }}\right.$ or $\left.\Delta D_{\text {inf }}\right)$ by that at the centre $\left(\Delta D_{\text {mid }}\right)-$ that is,

$$
\begin{aligned}
& \%_{\text {sup }}=100 \frac{\Delta \mathrm{D}_{\text {sup }}}{\Delta \mathrm{D}_{\text {mid }}}=100 \frac{\mathrm{D}_{\text {sup }}^{50}-\mathrm{D}_{\text {sup }}^{5}}{\mathrm{D}_{\text {mid }}^{\text {9 }}-\mathrm{D}_{\text {mid }}^{5}} \\
& \%_{\text {inf }}=100 \frac{\Delta \mathrm{D}_{\text {inf }}}{\Delta \mathrm{D}_{\text {mid }}}=100 \frac{\mathrm{D}_{\text {inf }}^{50}-\mathrm{D}_{\text {inf }}^{5}}{\mathrm{D}_{\text {mid }}^{\text {so }}-\mathrm{D}_{\text {mid }}^{5}}
\end{aligned}
$$

where the superscripts 5 and 50 refer to displacements at 5 and $50 \mathrm{~mm} \mathrm{Hg}$, respectively. All measured quantities were analysed statistically using a paired $t$ test.

\section{Results}

A low magnification overview of the $\mathrm{ONH}$ region from an eye fixed at $5 \mathrm{~mm} \mathrm{Hg}$ illustrates the nearly complete absence of posterior bowing in the LC (Fig 2), which may be compared with the pronounced posterior bowing of the LC in an eye at $50 \mathrm{~mm} \mathrm{Hg}$ (Fig 3). Despite the obvious difference in the posterior displacement, the LC does not appear to be thinner at $50 \mathrm{~mm} \mathrm{Hg}$ $\left(\mathrm{T}_{\text {avg }}=120.8 \mu \mathrm{m}\right)$ than at $5 \mathrm{~mm} \mathrm{Hg}$ $\left(T_{\text {avg }}=122.0 \mu \mathrm{m}\right)$ when comparing these two photomicrographs.

A higher magnification view of the LC region from an eye fixed at $5 \mathrm{~mm} \mathrm{Hg}$ demonstrates neatly aligned and nearly continuous vertical 'columns' of axonal bundles passing through the LC (Fig 4), which again may be compared with an eye fixed at $50 \mathrm{~mm} \mathrm{Hg}$ (Fig 5). Though there are some areas where the axonal columns are arranged into bundles, these columns are often disrupted by horizontal 'streaks' of what appear to be laminar connective tissue stretching across a number of axonal bundles in the coronal plane. 


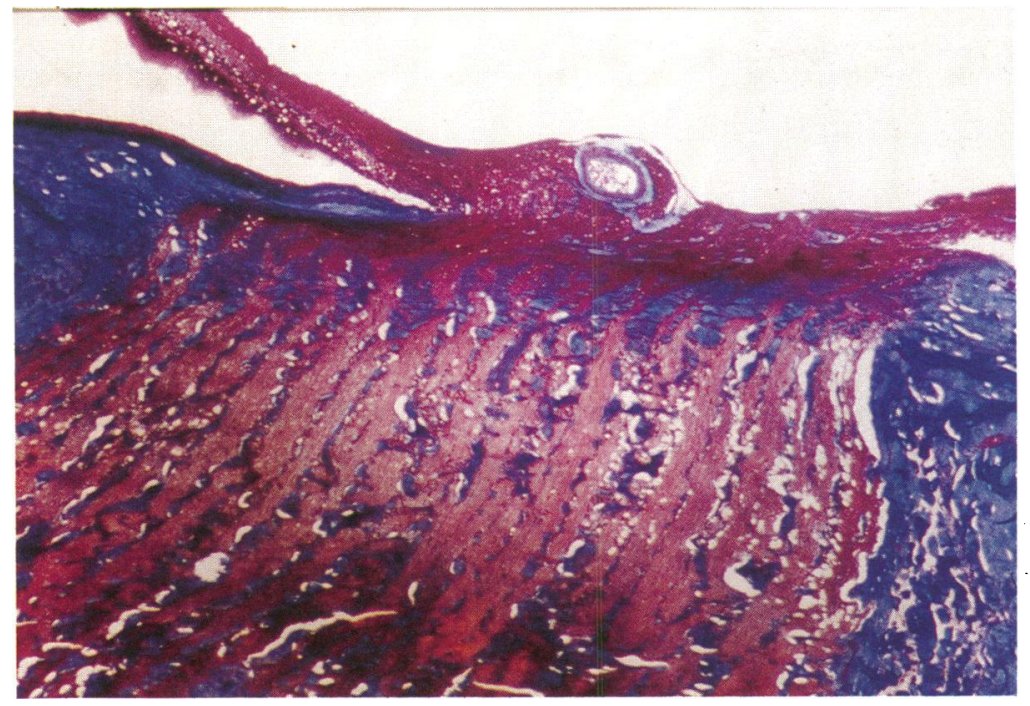

Figure 2 Overview of the optic nerve head region from a normal human eye fixed at $5 \mathrm{~mm} \mathrm{Hg}$. Note absence of posterior bowing of the lamina cribrosa (Gomori's trichrome, $\times 35)$.

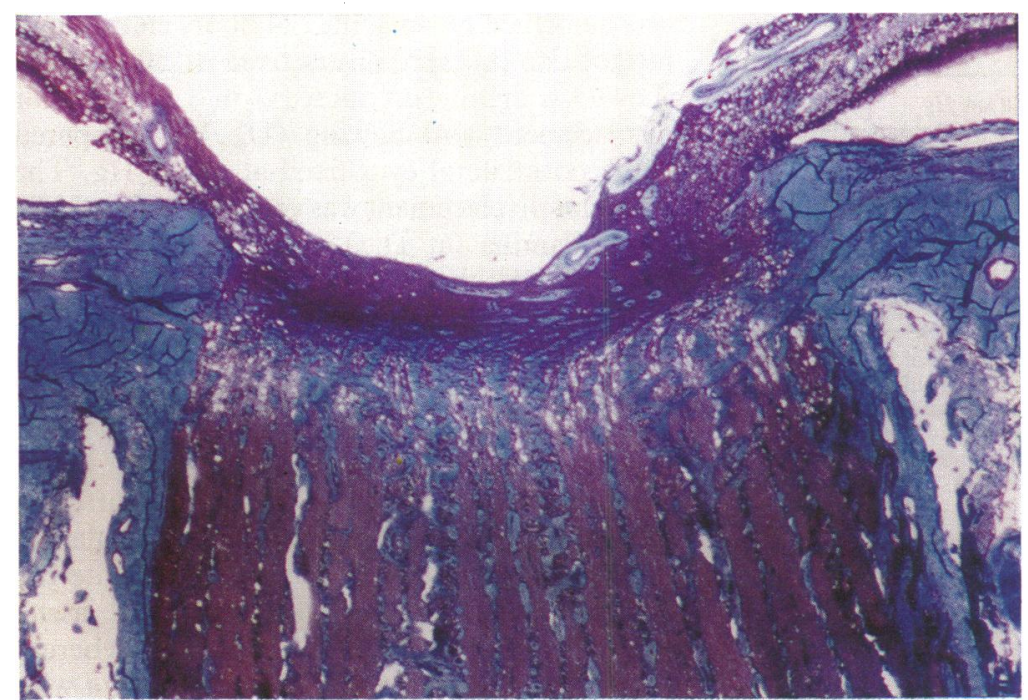

Figure 3 Overview of the optic nerve head region from a normal human eye fixed at $50 \mathrm{~mm} \mathrm{Hg}$. Note the marked posterior bowing but similar thickness of lamina cribrosa compared with Figure 2 (Gomori's trichrome, $\times 35$ ).

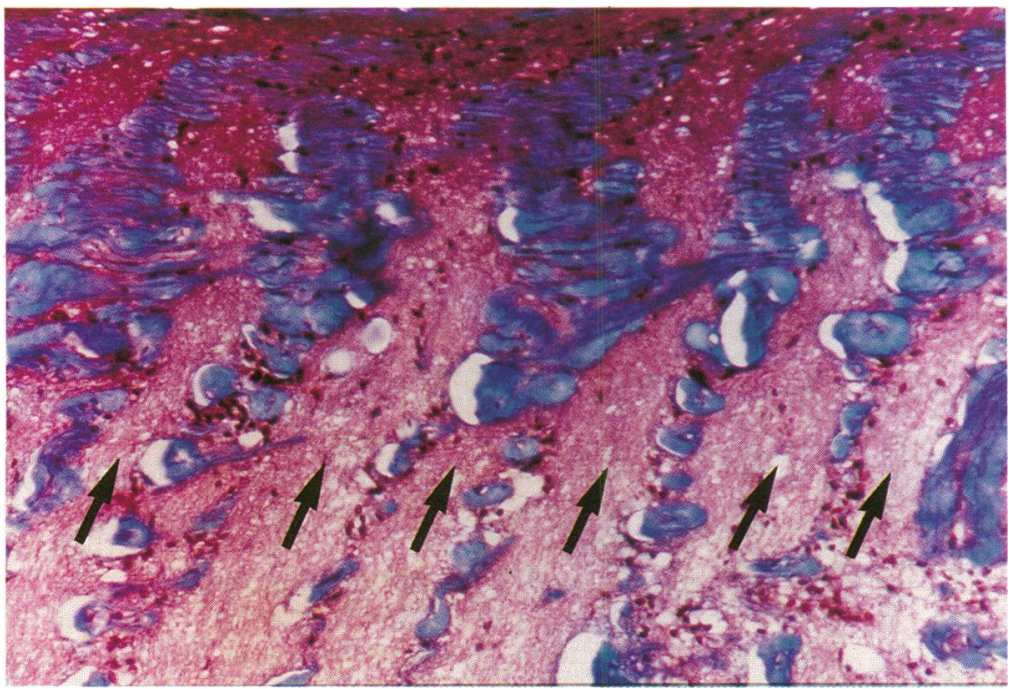

Figure 4 Lamina cribrosa of normal human eye fixed at $5 \mathrm{~mm} \mathrm{Hg}$. Axonal bundles traversing the lamina cribrosa show little interruption. Arrows: axonal bundles (Gomori's trichrome, $\times 140)$.
Although seen at $5 \mathrm{~mm} \mathrm{Hg}$ (for example, Fig 4), this pattern of streaking was more frequently observed and markedly more pronounced at $50 \mathrm{~mm} \mathrm{Hg}$ (for example, Fig 5). Moreover, this pattern of axonal columnar disruption was more commonly observed in the peripheral regions of the lamina.

The results of the morphometric analysis of the LC are summarised in Table 1 , while the characteristics of the donors are shown in Table 2. The width (W) of the LC at $50 \mathrm{~mm} \mathrm{Hg}$ was not significantly different from that at $5 \mathrm{~mm} \mathrm{Hg}$ $(\mathrm{p}=0.793)$, indicating that the increased pressure did not appreciably increase the size of the scleral canal through which the optic nerve passes. Posterior displacement $\left(D_{\text {mid }}\right)$ and normalised displacement $\left(\mathrm{D}_{\text {mid }} / \mathrm{W}\right)$ were significantly greater at $50 \mathrm{~mm} \mathrm{Hg}$ than at $5 \mathrm{~mm} \mathrm{Hg}$ $(\mathrm{p}=0.016$ and $\mathrm{p}=0.007$, respectively), with eight out of 10 pairs showing greater bowing $\left(D_{\text {mid }} / \mathrm{W}\right)$ at $50 \mathrm{~mm} \mathrm{Hg}$ than at $5 \mathrm{~mm} \mathrm{Hg}$ (Table 2). Posterior bowing was present in all high pressure eyes at least to some degree, with the minimum amount in the group being 0.074 However, only eyes fixed at the lower pressure showed little or no posterior displacement, though both the posterior displacement $(p=0.017)$ and bowing $(p=0.014)$ were significantly greater than zero in the low pressure group.

In order to determine if the observed morphometric findings were correlated with age, or if they were due to degenerative effects, morphometric measurements were linearly regressed against each of: patient age, postmortem time to enucleation, and postmortem time to fixation. No statistically significant correlation was found.

In order to analyse the topography of the LC, the profile of the anterior edge of the LC is plotted for 5 and $50 \mathrm{~mm} \mathrm{Hg}$ in Figure 6. In this figure, note that the displacement is by definition zero at the superior and inferior margins where the LC meets the sclera. The percentage of the total posterior displacement occurring in the superior quarter (that is, from 1 to 2 in Fig 6) was $\%_{\text {sup }}=69 \%(10 \%)$ mean (SE), and in the inferior quarter (that is, from 4 to 5 in Fig 6) was $\%_{\text {inf }}=66 \%(13 \%)$. Thus, approximately two thirds of the total deformation from 5 to $50 \mathrm{~mm} \mathrm{Hg}$ occurred in the periphery of the LC, while only one third occurred centrally.

The average thickness of the LC $\left(T_{\text {avg }}\right)$ at $50 \mathrm{~mm} \mathrm{Hg}$ was not significantly different from $\mathrm{T}_{\text {avg }}$ at $5 \mathrm{~mm} \mathrm{Hg}(\mathrm{p}=0.136$; paired $t$ test $)$. Moreover, LC thickness at the individual measurement locations $\left(T_{\text {sup }}, T_{\text {mid }}, T_{\text {inf }}\right)$ were not statistically different when compared with the two fixation pressures. Also, the thickness of the LC did not vary significantly across its width at $5 \mathrm{~mm} \mathrm{Hg}\left(\mathrm{p}=0.150\right.$ for $\mathrm{T}_{\text {sup }} v \mathrm{~T}_{\text {mid }} ; \mathrm{p}=0.198$ for $\left.\mathrm{T}_{\text {mid }} v \mathrm{~T}_{\text {inf }}\right)$ or at $50 \mathrm{~mm} \mathrm{Hg}\left(\mathrm{p}=0.813\right.$ for $\mathrm{T}_{\text {sup }} v$ $\mathrm{T}_{\text {mid }} ; \mathrm{p}=0.642$ for $\left.\mathrm{T}_{\text {mid }} v \mathrm{~T}_{\text {inf }}\right)$.

To assess the degree of correlation between eyes in a pair, LC thickness from eyes fixed at $5 \mathrm{~mm} \mathrm{Hg}$ is plotted against LC thickness from contralateral eyes fixed at $50 \mathrm{~mm} \mathrm{Hg}$ (Fig 7). The slope of a least squares linear regression fit to the data was close to 1 , indicating that the thickness of the lamina cribrosa correlated well between eyes of a given pair, despite the difference in fixation pressure. 


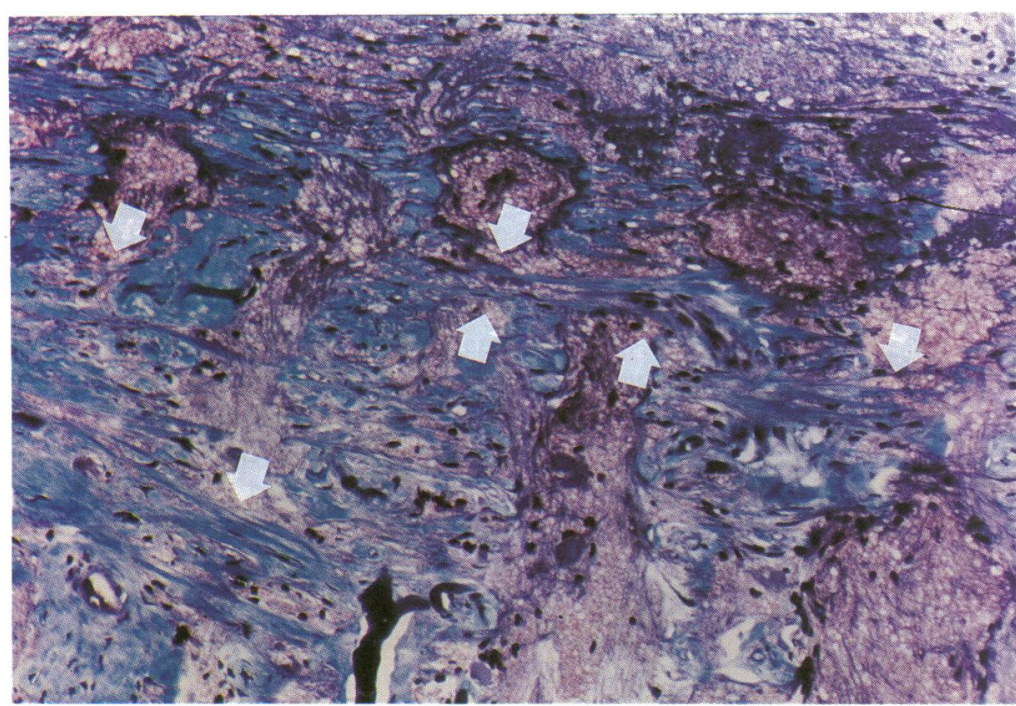

Figure 5 Lamina cribrosa of normal human eye fixed at $50 \mathrm{~mm} \mathrm{Hg}$. There is irregular disruption of axonal bundles and 'streaking' of the plates of the lamina cribrosa. Arrows. apparent interruption of axonal bundles (Gomori's trichrome, $\times 140$ ).

Table 1 Results of morphometric analysis of micrographs of the lamina cribrosa $(L C)$ from 10 pairs of normal human eyes fixed at either 5 or $50 \mathrm{~mm} \mathrm{Hg}$

\begin{tabular}{|c|c|c|c|}
\hline Measurement & $5 \mathrm{~mm} \mathrm{Hg}$ & $50 \mathrm{~mm} \mathrm{Hg}$ & p Value \\
\hline $\mathrm{W}(\mu \mathrm{m})$ & $1207(176)$ & $1197(163)$ & 0.793 (ns) \\
\hline $\mathrm{D}_{\mathrm{mid}}(\mu \mathrm{m})$ & $59(64)$ & $138(38)$ & 0.016 \\
\hline $\mathrm{D}_{\operatorname{mid}} \mathrm{w}$ & $0.050 \quad(0.050)$ & $0.114(0.028)$ & 0.007 \\
\hline $\mathrm{T}_{\mathrm{avg}}(\mu \mathrm{m})$ & $119(29)$ & $111(29)$ & $0 \cdot 136$ (ns) \\
\hline $\mathrm{T}_{\text {sup }}(\mu \mathrm{m})$ & $115(29)$ & $111(31)$ & 0.655 (ns) \\
\hline $\mathbf{T}_{\mathrm{mid}}(\mu \mathrm{m})$ & $124(34)$ & $110(29)$ & 0.065 (ns) \\
\hline $\mathbf{T}_{\mathrm{inf}}(\mu \mathrm{m})$ & $118(27)$ & $112(31)$ & 0.351 (ns) \\
\hline
\end{tabular}

$\mathrm{W}=$ width of $\mathrm{LC} ; \mathrm{D}=$ posterior displacement of $\mathrm{LC} ; \mathrm{T}=$ thickness of LC. Column $4, p$ is a probability value calculated using a paired two sided Student's $t$ test using measurements averaged between three observers. ns=not statistically significant. See Figure 1 and text for more complete definition of measured quantities. Values tabulated are mean (SD).

Table 2 Pairwise comparison of normalised posterior displacement $\left(D_{\text {mid }} / W\right)$ measured in 10 pairs of normal human eyes at 5 and $50 \mathrm{~mm} \mathrm{Hg}$, as described in text

\begin{tabular}{lllll}
\hline Pair $($ no $)$ & Age (years) & Sex & $D_{\text {mid }} /$ at $5 \mathrm{~mm} \mathrm{Hg}$ & $D_{\text {mid }} /$ at $50 \mathrm{~mm} \mathrm{Hg}$ \\
\hline 1 & 75 & M & 0.036 & $0 \cdot 129$ \\
2 & 85 & M & 0.024 & $0 \cdot 137$ \\
3 & 81 & F & 0.031 & 0.074 \\
4 & 69 & M & 0.001 & $0 \cdot 126$ \\
5 & 46 & M & 0.131 & $0 \cdot 105$ \\
6 & 59 & F & 0.071 & 0.093 \\
7 & 64 & F & 0.029 & $0 \cdot 124$ \\
8 & 84 & 0.073 & 0.079 \\
9 & 88 & M & -0.023 & $0 \cdot 168$ \\
10 & 39 & & & 0.119 \\
\hline
\end{tabular}

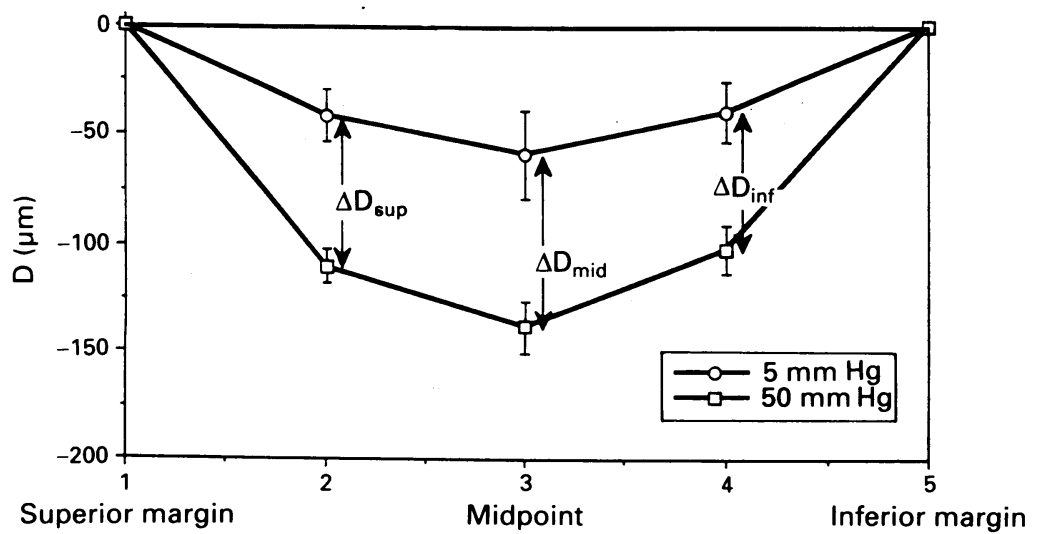

Figure 6 Plot of the location of the anterior surface of the $L C$ in high and low pressure groups of eyes. Values shown are mean with standard error bars. The posterior displacement is taken to be zero, by definition, at the superior and inferior margins where the $L C$ inserts into the sclera. Note also the terminology defining $\Delta D$ at the superior, midpoint, and inferior locations.

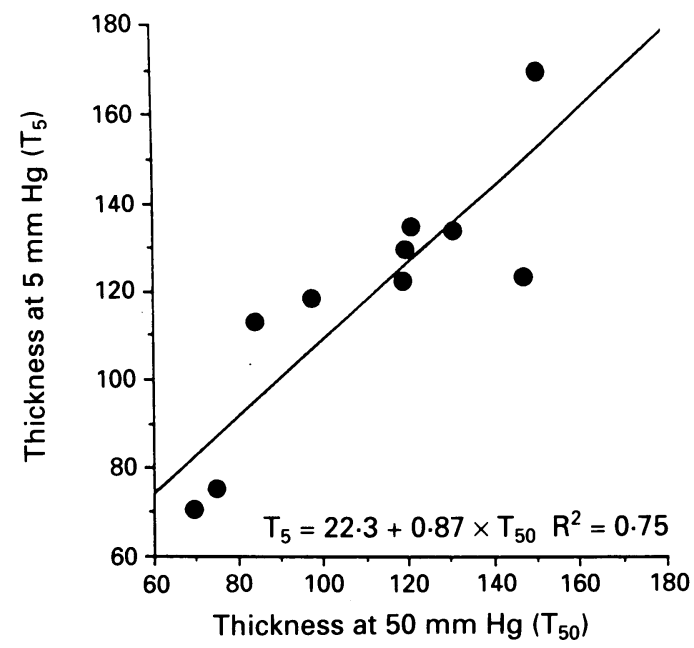

Figure 7 Plot of LC thickness at $50 \mathrm{~mm} \mathrm{Hg}$ v LC thickness at $5 \mathrm{~mm} \mathrm{Hg}$ for pairs of eyes used in the present study. The line was obtained by linear least squares regression.

\section{Discussion}

In enucleated human eyes, we found that the LC was deformed by an acute ( 24 hour) elevation of intraocular pressure. Eyes fixed at $50 \mathrm{~mm} \mathrm{Hg}$ showed a significant increase in LC posterior displacement and bowing $\left(\mathrm{D}_{\text {mid }} / \mathrm{W}\right)$ compared with contralateral eyes fixed at $5 \mathrm{~mm} \mathrm{Hg}$. The posterior displacement was greatest in the middle of the lamina (at $\mathrm{D}_{\text {mid }}$ ), such that the lamina assumed a ' $U$ ' shape. This finding agrees qualitatively with that of Levy et al, ${ }^{11}$ although the average posterior displacement $\left(D_{\text {mid }}\right)$ caused by a rise in IOP from 5 to $50 \mathrm{~mm} \mathrm{Hg}$ was quantitatively much greater in the present study $(79 \mu \mathrm{m})$ than in that of Levy et $a l^{11}(26 \mu \mathrm{m})$. This discrepancy may be due in part to the different methods used in the two studies; in particular, in the present study we were able to estimate the displacement of a clearly identified tissue boundary (anterior face of the LC), whereas Levy et al measured the displacement of a wire presumably moving with the tissue mass into which it was inserted.

Although the finding that bowing increased from 5 to $50 \mathrm{~mm} \mathrm{Hg}$ is noteworthy, it may be equally important that the LC at low pressure is not actually flat. In fact, there was approximately $44 \%$ as much bowing at $5 \mathrm{~mm} \mathrm{Hg}$ as there was at $50 \mathrm{~mm} \mathrm{Hg}$, indicating that an appreciable amount of LC deformation occurs at normal or subnormal IOPs. Interestingly, there was much greater variability in the amount of bowing present in the low pressure eyes than in the high pressure group; in fact, four out of the 10 eyes in the low pressure group showed bowing which fell into the range for the high pressure group (Table 2). However, there was appreciable variability in bowing even in the eyes at high pressure. This may reflect normal interpatient variability, and we speculate that eyes with less bowing at high pressure may be at lower risk of developing optic neuropathy under conditions of ocular hypertension. We conclude that the response of the LC is more variable at lower pressures, and some LC seem to be prone to bowing at normal or subnormal IOPs. It would be of interest to investigate this phenomenon further, since a significant number of glaucoma patients with progressive 
optic neuropathy actually have normal IOPs (low tension glaucoma).

Although there was a weak trend towards thinning of the LC from 5 to $50 \mathrm{~mm} \mathrm{Hg}$ at the midpoint, this result was not statistically significant. We conclude that the thickness of the LC was not appreciably affected by the elevation of intraocular pressure. The experimental precision of our measurements of LC thickness was supported by the excellent correlation in LC thickness between eyes of a given pair, despite the greater than twofold range in LC thickness observed among all the pairs. We conclude that bowing of the LC resulted from the posterior movement of the sheets of the LC in unison, thus preserving the overall LC thickness. These findings contrast with those of Quigley, ${ }^{3}$ who correlated the clinical severity of the glaucoma to the shape of the LC when fixed at zero pressure. In that study, it was found that the thickness of the LC progressively decreased with the severity of the glaucoma, and that the LC assumed the shape of a 'W' such that $D_{\text {mid }}$ was less than $D_{\text {sup }}$ and $D_{\text {inf }}$ in the group of eyes with the most severe glaucoma. Since we found that increased intraocular pressure did not immediately decrease the thickness of the lamina, we postulate that thinning of the lamina is a chronic effect occurring in glaucomatous optic neuropathy.

From the above observations, it is possible to make several deductions about the stress distribution within the LC. In our experiments, a pressure difference of either 5 or $50 \mathrm{~mm} \mathrm{Hg}$ existed between the anterior surface of the $\mathrm{ONH}$ and the retrolaminar region of the optic nerve.

The distribution of this pressure drop in the

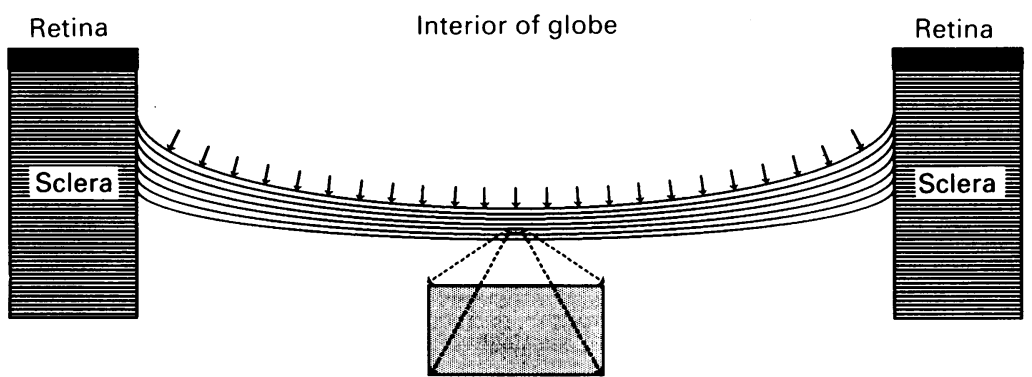

Figure 8 Schematic diagram of a possible mode of deflection of the lamina cribrosa caused by elevataed IOP. In this diagram, the majority of the pressure drop occurs near the anterior face of the LC. Note the thinning of the $L C$, with attendant tissue compression concentrated near the centre of the $L C$.

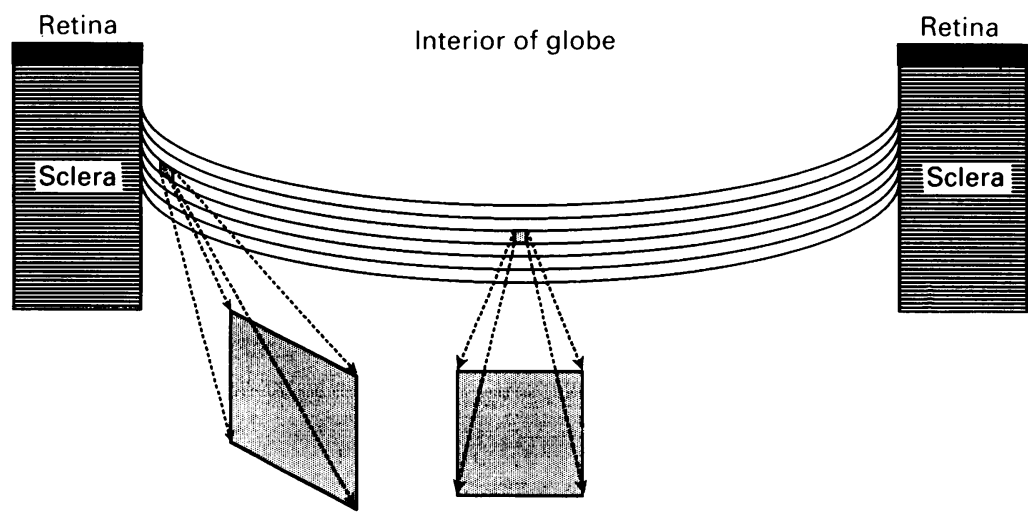

Figure 9 Schematic diagram of a possible mode of deflection of the lamina cribrosa caused by elevated IOP. In this diagram, the pressure drop is distributed across the LC. In this case the thickness of the $L C$ is not significantly affected. Also shown are two tissue elements in the deflected condition. Note the pronounced shearing at the periphery of the lamina, leading to the skewed shape of the tissue element. intervening tissue determines the loads imposed on the LC and the nature of the resulting deformations. Consider first a model in which the pressure drop occurs at the anterior surface of the LC, caused by sealing of the globe at the $\mathrm{ONH}$ by the innermost sheets of the lamina. The resulting pressure difference would displace the anterior sheets of the LC posteriorly into the sheets behind them, implying that the LC should become thinner under pressure (Fig 8). Specifically, the model predicts that the central region of the LC should be maximally thinned, while the thickness of the peripheral LC inserting into the relatively incompressible sclera should change little from 5 to $50 \mathrm{~mm} \mathrm{Hg}$. Such a pattern of LC thinning was not observed in this study. In particular, LC thickness at the midpoint $\left(\mathrm{T}_{\mathrm{mid}}\right)$ was not significantly different from LC thickness at the periphery $\left(T_{\text {inf }}, T_{\text {sup }}\right)$. We conclude that this model is incorrect. A second scenario, which is supported by this study, is that the pressure drop across the lamina is distributed across the total anteroposterior thickness of the lamina. This distribution of pressure drop throughout the LC will yield tissue loading conditions causing little overall change to the thickness of the lamina (Fig 9). Such a distributed pressure drop is most consistent with the slow posterior flow of a small amount of fluid through the lamina, such as occurs in all connective tissues to some extent. ${ }^{16}$ Such leakage will cause a net distributed force in the direction of fluid flow - that is, in the anteroposterior direction, as shown in Figure 9.

We observed in this study that most of the posterior displacement due to pressure elevation occurred within the peripheral areas of the LC, while the thickness of the LC did not change. These observations are consistent with a mechanical model in which shear forces within the LC dominate compressive or tensile stresses, just as occurs in the deflection of a thick flat disc supported at its edge ${ }^{17}$ which is subjected to a net force in the anteroposterior direction. In such a model, shear stresses are maximal at the border between the sclera and the LC, and decrease as one moves away from the scleral boundary until they are negligible at the centre of the LC. The geometric interpretation of this behaviour is schematically illustrated in Figure 9, in which two elements of tissue in the LC are shown in their deformed states. Both tissue elements are square at low pressure; however, the element at the periphery of the LC undergoes more shearing deformation than does the centrally located element, owing to the greater curvature at the periphery.

This shear stress model is also consistent with clinically observed patterns of visual field loss in glaucoma. Although early patterns of damage are variable, it is generally accepted that the very centre of the visual field is usually the last to be affected in glaucoma, ${ }^{18}$ and that the central visual field is supplied by nerve fibre bundles which pass more centrally through the LC than do fibres emanating from the peripheral retina. ${ }^{19}$ Thus, nerve fibre bundles least affected in glaucoma pass through the LC in a region where tissue deformation and shear stresses are relatively small compared with those in the peripheral lamina. This is in contradistinction to the 
distribution of compressive stresses, which increase as one moves centrally within the $\mathrm{ONH}$.

Since only one section of the $\mathrm{ONH}$ per eye was examined in this study, it was not possible to determine if increased IOP preferentially deforms the LC in the superior and inferior regions of the ONH. Thus, the present study did not address correlations between the characteristic paracentral scotomas and arcuate visual field defects seen in early glaucoma and the relative paucity of connective tissue in the superior and inferior poles of the LC. ${ }^{2021}$ This issue could be addressed in a further study in which serial sections of the $\mathrm{ONH}$ were measured to form a three dimensional reconstruction of the LC region.

The fine structure of the $\mathrm{ONH}$ was also altered by the acute elevation of pressure, the most notable finding being the disruption of the axonal bundles within the LC. It is unlikely that these changes were solely due to postmortem artefacts, since they occurred to a much greater extent in high pressure eyes than in contralateral low pressure eyes. Further, no correlation between the presence of axonal bundle disruption and the postmortem fixation time of the eyes was observed. It is possible that the observed disruption of axonal bundles could be caused by bundles coming in and out of the plane of section as they traverse the LC. However, this explanation is inconsistent with the observation that the LC was not thinned in high pressure eyes, since thinning of the $\mathrm{LC}$ would be required to increase the tortuosity of the nerve fibre bundles.

In conclusion, acute elevation of IOP causes the LC to bow posteriorly without affecting its thickness. Such a change to the shape of the lamina is consistent with a model of force distribution within the LC in which shear stresses are dominant. In addition, the shear stress model is consistent with the clinical finding that the central visual field is least vulnerable to glaucomatous damage, since this area of the visual field is supplied by nerve fibre bundles which enter the globe through the central area of the ONH where shear stresses should be minimal. Such deformation of the LC could disrupt axonal bundles of the optic nerve as they traverse the lamina, as was observed in some of the high pressure eyes we examined. These findings support a model of early mechanical damage to the optic nerve in which shear stresses are an important pathogenic mechanism in the aetiology of optic nerve fibre bundle loss.

This material was presented in part at the 1992 ARVO Meeting, Sarasota, FL, USA. Supported by MRC Grant MA-10051 and funds from the Glaucoma Research Society of Ontario.

1 Emery JM. The lamina cribrosa in normal and glaucomatou human eyes. Symposium on Glaucoma 1974; 78: 290-7.

2 Quigley HA, Addicks EM, Green WR, Maumenee AE. Optic nerve damage in human glaucoma; II: The site of injury and susceptibility to damage. Arch Ophthalmol 1981;99: 635-49.

3 Quigley HA. Morphological changes in the lamina cribrosa correlated with neural loss in open-angle glaucoma. $A m \mathcal{F}$ Ophthalmol 1983; 95: 673-81.

4 Quigley HA, Anderson DR. The dynamics and location of axonal transport blockade by acute intraocular pressure elevation in primate optic nerve. Invest Ophthalmol Vis $S c i$ 1976; 15: 606-16.

5 Minckler DS, Bunt AH, Klock IB. Radioautographic and cytochemical ultrastructural studies of axoplasmic transport in the monkey optic nerve head. Invest Ophthalmol Vis $S c i$ 1978; 717: 33-50.

6 Anderson DR, Hendrickson A. Effect of intraocular pressure on rapid axonal transport in monkey optic nerve. Invest Ophthalmol Vis Sci 1974; 13: 771-83.

7 Quigley HA, Flower RW, Addicks EM, McLeod DS. The mechanism of optic nerve damage in experimental acute mechanism of optic nerve damage in experimental acute intraocular pressure

8 Maumenee AE. Causes of optic nerve damage in glaucoma. Ophthalmology 1983; 90: 741-52.

9 Zeimer RC, Ogura Y. The relation between glaucomatous damage and optic nerve head mechanical compliance. Arch Ophthalmol 1989; 107: 1232-4.

10 Coleman AL, Quigley HA, Vitale S, Dunkelberger MS. Displacement of the optic nerve head by acute changes in intraocular pressure in monkey eyes. Ophthalmology 1991; 98: $37-40$.

11 Levy NS, Crapps EE. Displacement of optic nerve head in response to short-term intraocular pressure elevation in response to short-term intraocular pressure el
human eyes. Arch Ophthalmol 1984; 102:782-6.

12 Levy NS, Crapps EE, Bonney RC. Displacement of the optic nerve head: response to acute intraocular pressure elevation in primate eyes. Arch Ophthalmol 1981; 99: 2166-74.

13 Nesterov AP, Egorov EA. Pathological physiology of primary open angle glaucoma. In: Cairns J, ed. Glaucoma. Miami: Grune and Stratton, 1986: 382-93.

14 Johnson DH, Tschumper RC. Human trabecular meshwork organ culture: a new method. Invest Ophthalmol Vis $\mathrm{Sci}$ 1987; 28: 945-53.

15 Maynard JH. A trichrome stain in glycol methacrylate that works. Lab Med 1986; 17: 471-3.

16 Levick JR. Flow through interstitium and other fibrous matrices. $Q \mathcal{F}$ Exp Physiol 1987; 72: 409-28.

17 Wang C-T. Applied elasticity. New York: McGraw Hill, 1953 . Section 11.5:291-6.

18 Drance SM. The glaucomatous visual field. Invest Ophthalmol Vis Sci 1972; 11: 85-97.

19 Minckler DS. The organization of nerve fibre bundles in the primate optic nerve head. Arch Ophthalmol 1980; 98 . 1630-6.

20 Quigley HA, Addicks EM. Regional differences in the structure of the lamina cribrosa and their relation to glaucomatous optic nerve damage. Arch Ophthalmol 1981; 99: 137-43.

21 Radius RL, Ganzales $M$. Anatomy of the lamina cribrosa in human eyes. Arch Ophthalmol 1981; 99: 2159-62. 